\title{
Day-to-day associations between subjective sleep and affect in regard to future depression in a female population-based sample
}

Jessica A. de Wild-Hartmann, Marieke Wichers, Alex L. van Bemmel, Catherine Derom, Evert Thiery, Nele Jacobs, Jim van Os and Claudia J. P. Simons

\section{Background}

Poor sleep is a risk factor for depression, but little is known about the underlying mechanisms.

\section{Aims}

Disentangling potential mechanisms by which sleep may be related to depression by zooming down to the 'micro-level' of within-person daily life patterns of subjective sleep and affect using the experience sampling method (ESM).

\section{Method}

A population-based twin sample consisting of 553 women underwent a 5-day baseline ESM protocol assessing subjective sleep and affect together with four follow-up assessments of depression.

\section{Results}

Sleep was associated with affect during the next day, especially positive affect. Daytime negative affect was not associated with subsequent night-time sleep. Baseline sleep predicted depressive symptoms across the follow-up period.

\section{Conclusions}

The subtle, repetitive impact of sleep on affect on a daily basis, rather than the subtle repetitive impact of affect on sleep, may be one of the factors on the pathway to depression in women.

\section{Declaration of interest}

J.V.O. is or has been an unrestricted research grant holder with, or has received financial compensation as an independent symposium speaker from, Eli Lilly, Bristol-Myers Squibb, Lundbeck, Organon, Janssen-Cilag, GlaxoSmithKline, Astrazeneca, Pfizer and Servier.
Sleep and affect are closely intertwined. This link is most evident in depression, where a large body of epidemiological studies has shown that sleep disturbances appear to be a risk factor for - as well as a symptom of - depression (e.g. Ohayon \& Roth, ${ }^{1}$ Cho et $a l^{2}$ for an overview see Baglioni et $\left.a l^{3}\right)$.

Longitudinal cohort studies investigating the association between sleep disturbances and depression (with years between the assessments) are very suited to demonstrating unbiased associations, but lend themselves less to unravelling the underlying mechanisms contributing to these associations. To identify principles underlying the link between sleep and depression, the focus should be narrowed down to the daily associations of sleep and affect.

The experience sampling method (ESM), an ambulatory diary technique assessing variables of interest prospectively and repeatedly across the day for a period of time, represents a suitable approach to zoom into the daily within-person associations between subjective sleep and affect. ${ }^{4,5}$ In addition, its prospective character allows for the examination of directions of daily sleep-affect associations, an aspect which is, with two exceptions, ${ }^{6,7}$ largely disregarded.

The purpose of the present study was to disentangle potential mechanisms by which sleep may be related to depression by (1) zooming down to the 'micro-level' of within-person daily life patterns of subjective sleep and affect using the ESM and (2) zooming out to the 'macro-level' of subjective sleep and affect by investigating the association between baseline sleep and future depression in a female population-based sample.

\section{Method}

\section{Sample}

The sample comprised 621 women (577 twins and 44 non-twin sisters) aged 18-61 years (mean 28 years). Participants were recruited from the East-Flanders Prospective Twin Survey (EFPTS $)^{8,9}$ and from birth registers of Flemish municipalities. The EFPTS is a population-based survey that started in 1964, registering prospectively all multiple births within East Flanders. Although most participants were twins, the current study did not require twin methodology. Originally, the study was designed to assess heritability of stress sensitivity in daily life, and given evidence for qualitative differences in the type of environmental stressors that are associated with depression in men and women, ${ }^{10,11}$ the sample was female only. The project was approved by the local medical ethics committee and all participants gave their written consent.

\section{Experience sampling method}

The ESM was used to assess daily life momentary affective states (Fig. 1). The ESM is a validated, structured diary technique to assess individuals in their daily living environment. ${ }^{12,13}$ Participants received a digital wristwatch and a set of ESM self-assessment forms collated in a booklet for each day. The wristwatch was programmed to emit a signal ('beep') at an unpredictable moment in each of ten 90-minute time blocks between 07:30 and 22:30 on 5 consecutive days. After each beep, participants were required to stop their activity and fill out the ESM self-assessment forms. All self-assessments were rated on 7-point Likert scales.

In addition to the self-assessments after each beep, participants were asked to complete a sleep questionnaire comprising an evening and morning part. The morning part had to be filled in immediately on awakening and the evening part immediately prior to sleep (see below).

Trained research assistants with ample experience in momentary assessment techniques explained the ESM procedure to the participants during an initial briefing session, and participants 


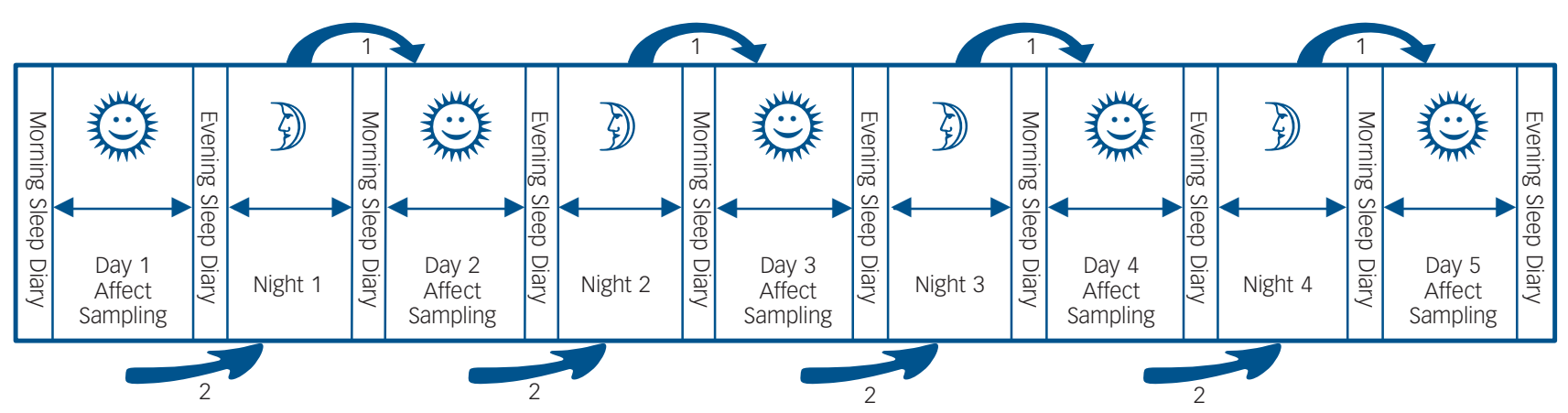

Fig. 1 Schematic overview of the study protocol.

For the duration of 5 consecutive days, participants rated positive and negative affect items ten times a day and filled in sleep questionnaires in the morning and evening. This design allows for the investigation of the association between sleep and subsequent daytime affect (arrow 1 in the figure), as well as affect during the day and subsequent sleep (arrow 2 in the figure).

completed an ESM practice form to confirm that they understood the 7-point Likert scale. Participants could call a telephone number in case they had questions or problems during the ESM sampling period. Participants were told to complete their reports immediately after the beep, thus minimising memory distortion, and to record the time at which they completed the form. To check whether the participants had completed the form within $15 \mathrm{~min}$ of the beep, the time at which participants indicated they completed the report was compared with the actual time of the beep. All reports not filled in within $15 \mathrm{~min}$ after the beep were excluded from the analysis, since previous work ${ }^{13}$ has shown that reports completed after this interval are less reliable and consequently less valid. In addition, previous work has shown that participants who have valid reports for at least a third of all measurements can be included since their missing data do not distort the results, whereas measures of individuals with fewer than $30 \%$ of completed reports are less reliable. ${ }^{13}$

\section{Procedure}

The study consisted of five parts: a baseline part $\left(T_{0}\right)$, in which the 5-day ESM study took place, and four follow-up assessments $\left(T_{1}-T_{4}\right)$. The average period between the interview assessments was 134 days for the period $T_{0}-T_{1}, 91$ days for the period $T_{1}-T_{2}$, 116 days for the period $T_{2}-T_{3}$ and 91 days for the period $T_{3}-T_{4}$.

\section{Measures of affect}

Using the ESM procedure at baseline as described above, participants rated their mood states by using ten adjectives on a 7-point Likert scale, ranging from 1 (not) to 7 (very). Factor analysis identified two mood states: positive affect and negative affect. Positive affect comprised the weighted average of ratings on 'cheerful', 'content', 'energetic' and 'enthusiastic'; negative affect comprised the weighted average of ratings on 'insecure', 'lonely', 'anxious', 'low', 'guilty' and 'suspicious'.

\section{Sleep measurements}

Subjective sleep was assessed by means of a sleep diary. A sleep diary is a way of prospectively self-monitoring sleep and is regarded as the gold standard for subjective sleep assessment. ${ }^{14}$ Furthermore, it allows specific investigation of within-person daily life patterns of subjective sleep and affect, the main aim of the current study.

Each morning, participants rated subjective sleep variables pertaining to the previous night: sleep quality on a 7-point Likert scale ranging from 1 (not at all) to 7 (very well), sleep onset latency (time taken to fall asleep, in minutes), and number of awakenings. Furthermore, participants reported the (clock-) time of awakening and getting up. Each evening, participants recorded their bedtime, enabling estimation of total time in bed (time in minutes between time to bed and getting up). In order to estimate total sleep time, the variable sleep period was generated, reflecting time in minutes between reported sleep time and awakening.

\section{Depressive symptomatology}

The Structured Clinical Interview for DSM-IV Axis I Disorders $(\mathrm{SCID}-\mathrm{I})^{15}$ was administered at baseline $\left(T_{0}\right)$ and at the fourth follow-up $\left(T_{4}\right)$. The SCID-I is a diagnostic instrument to assess the presence of DSM-IV Axis I disorders and was used to obtain current and lifetime diagnosis of major depressive disorder. In addition to the SCID-I, subclinical depressive symptomatology was assessed at baseline and at each follow-up using the Symptom Checklist-90-Revised (SCL-90-R). ${ }^{16}$ The SCL-90-R is a widely used 90 -item self-report questionnaire measuring general psychological distress. For the analyses, the SCL-90-R depression subscale was used, consisting of 13 items, rated on a 5-point Likert scale.

\section{Other measures}

During every daily affect rating within the ESM protocol, participants were asked about previous alcoholic or food intake (yes/no) and level of physical exercise (7-point Likert scale) since the last beep.

Furthermore, participants completed the NeuroticismExtraversion subscale of the Eysenck Personality Scale ${ }^{17}$ and rated their somatic health on a 5 -point Likert scale $(1=$ very well, $5=$ bad) at baseline.

\section{Analysis plan}

Two sets of analyses were conducted. The first investigated the day-to-day associations between sleep and affect, using data from the baseline ESM study. The second set of analyses investigated the longitudinal associations between baseline measures of sleep and depressive symptomatology at the follow-up assessments.

\section{ESM data associations between sleep and affect}

The baseline ESM data-set has a hierarchical structure, due to within-person clustering: repeated measurements (level 1) are nested within individuals (level 2) and individuals are nested within twin pairs (level 3). That is, observations from the same 
participant are more similar than observations from different participants, and observations within a twin pair are more similar than between twin pairs. As nesting of data violates the assumption of independent residuals, multilevel regression analysis was used that takes clustering (in this case within participants and within twin pairs) into account. ${ }^{18}$

Associations between (1) subjective sleep and ESM measures of affect the next day, and between (2) ESM daytime affect and subsequent sleep, were separately calculated using the XTMIXED command in STATA (version 11 for Windows) (Fig. 1). The first model included sleep as a predictor of affect the next day and was additionally corrected for food and alcohol intake, physical exercise and age. In this model, no aggregation took place and all data points were used (i.e. ten affect ratings per day for each individual). The second model included affect as a predictor of subsequent sleep and was additionally corrected for food and alcohol intake as well as physical exercise during the day. In this model, day aggregates of the independent variable (affect) were used (i.e. the mean of multiple measures of affect per day for each individual), as the dependent variable (sleep) was only measured once a day. Thus, in this model, repeated measurements over multiple days were clustered within participants and participants within twin pairs. Six day-of-the-week dummy variables were included in both models to account for daily variation (reference category Sunday). As we were only interested in the within-person component of the regression estimation, predictor variables were centred at the participant level. Regression coefficients were standardised with the aid of the STATA command ESTADD BETA. All regression analyses were controlled for age.

\section{Baseline sleep and follow-up depressive symptoms}

The associations between baseline subjective sleep and follow-up SCL-90-R depressive symptoms were analysed using multilevel regression analysis (STATA command XTMIXED), as multiple follow-up measurements $T_{1}-T_{4}$ (level 1 ) were clustered within participants (level 2), who were part of twin pairs (level 3). That is, the four follow-up measurements of depressive symptomatology $\left(T_{1}-T_{4}\right)$ were regressed on mean scores of the separate sleep variables. For the association between sleep at baseline and follow-up DSM-IV diagnosis of depression as response variable, multilevel logistic regression analyses were conducted using STATA command XTLOGIT, as participants are clustered within twin pairs. Participants with a current or lifetime diagnosis of major depression according to DSM-IV were excluded and analyses were controlled for age, baseline SCL-90-R depressive symptoms, trait neuroticism and somatic health. Regression coefficients were standardised with the aid of the STATA command ESTADD BETA.

\section{Results}

\section{Sample}

The total sample consisted of 621 White female participants, of whom 610 participated in the ESM procedure. Thirty-one women were excluded because they had fewer than 17 valid ESM selfreports or missing reports. A total of 26 participants were excluded because they fulfilled DSM-IV criteria for a major depressive episode at baseline, resulting in 553 participants with valid ESM measures at baseline. See Table 1 for a summary of demographic variables, sleep variables and depressive symptoms at baseline. The number of participants varied slightly across variables owing to partial missing data (Table 1).

For the longitudinal part of the study, that is, the association between baseline sleep and future depression, 82 participants were

\begin{tabular}{|c|c|c|c|}
\hline & Mean & s.d. & $n$ \\
\hline \multicolumn{4}{|l|}{ Demographics } \\
\hline Age, years & 27.77 & 7.86 & 551 \\
\hline Education $^{a}$ & 3.26 & 1.02 & 552 \\
\hline Employed, \% & 61.20 & & 551 \\
\hline Married, \% & 35.96 & & 551 \\
\hline \multicolumn{4}{|l|}{ Sleep characteristics } \\
\hline Sleep quality ${ }^{b}$ & 5.26 & 1.46 & 553 \\
\hline Sleep onset latency, ${ }^{c}$ minutes & 21.40 & 26.85 & 553 \\
\hline Sleep period, ${ }^{\mathrm{d}}$ minutes & 471.03 & 87.07 & 531 \\
\hline Number of awakenings & 1.5 & 1.76 & 553 \\
\hline \multicolumn{4}{|l|}{ Affect, weighted average } \\
\hline Positive & 3.57 & 1.00 & 541 \\
\hline Negative & 0.82 & 0.35 & 553 \\
\hline \multicolumn{4}{|l|}{ Depressive symptoms } \\
\hline SCL-90-R baseline score ${ }^{e}$ & 1.41 & 0.45 & 548 \\
\hline SCL-90-R follow-up score, mean aggregated & 1.41 & 0.50 & 440 \\
\hline SCID-I depression at $T_{4}, \%$ & 2.7 & & 362 \\
\hline \multicolumn{4}{|c|}{$\begin{array}{l}\text { SCL-90-R, Symptom Checklist-90-Revised; SCID-I, Structured Clinical Interview for } \\
\text { DSM-IV Axis I Disorders. } \\
\text { a. Assessed on a 4-point scale: } 1=\text { primary school; } 4=\text { = university degree. } \\
\text { b. Rated on a 7-point Likert scale: } 1=\text { not at all, } 7=\text { very well. } \\
\text { c. Time needed to fall asleep. } \\
\text { d. Time between falling sleep and awakening (without considering nightly awakenings) } \\
\text { e. Rated on 5-point Likert scale. }\end{array}$} \\
\hline
\end{tabular}

additionally excluded because they fulfilled DSM-IV criteria for current or lifetime diagnosis of depression, resulting in 471 participants. Data with respect to follow-up SCL-90-R depressive symptoms were present for 423 participants at $T_{1}, 417$ at $T_{2}, 392$ at $T_{3}$ and 383 at $T_{4}$. For 440 participants, there was at least one follow-up assessment of the SCL-90-R. Thirty-one participants dropped out for the entire follow-up period. They did not differ significantly in terms of baseline sleep quality $(t=-0.39$, $P=0.70)$ or baseline depressive symptomatology $(t=0.73$, $P=0.47$ ). Data with respect to follow-up SCID-I current depressive episode at $T_{4}$ were available for 362 participants. Drop-out for the SCID-I at $T_{4}$ was not associated with baseline sleep quality $(t=-0.69, \quad P=0.48)$ or baseline depressive symptomatology $(t=-1.26, P=0.21)$.

\section{Subjective sleep as a predictor for affect the next day} Positive affect

There was a significant association between all sleep variables and positive affect the next day. Sleep quality and sleep period showed a positive linear association with positive affect $(\beta=0.06$, $P<0.001$ and $\beta=0.02, P=0.011$ respectively), whereas number of awakenings and sleep latency showed a negative association ( $\beta=-0.01, P=0.009$ and $\beta=-0.01, P=0.049$ respectively). The association between sleep quality and positive affect yielded the highest effect size (Fig. 2).

\section{Negative affect}

There was a significant negative association between sleep quality and negative affect $(\beta=-0.03, P<0.001)$ and a significant positive association between sleep latency and negative affect ( $\beta=0.01, P=0.028)$, but not between negative affect and sleep period $(\beta<0.01, P=0.532)$, or number of awakenings $(\beta<0.01$, $P=0.828)$.

\section{Affect during the day as a predictor for sleep Positive affect}

Sleep quality was negatively associated with prior daytime positive affect $(\beta=-0.05, P=0.008)$. Sleep latency $(\beta<-0.01, P=0.826)$, 


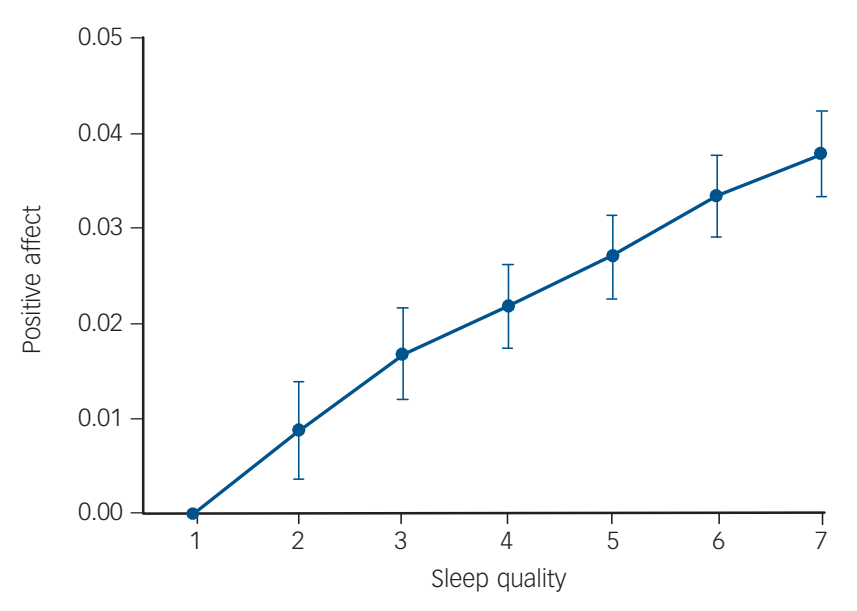

Fig. 2 Effect sizes of subjective sleep quality as reported on awakening on average positive affect during the day: increase of positive affect per category of sleep quality $(1=$ very bad, $7=$ very good) relative to reference category sleep quality $=1$.

sleep period $(\beta<-0.01, P=0.889)$ and number of awakenings ( $\beta=0.03, P=0.095)$ showed no significant association with prior positive affect.

\section{Negative affect}

Sleep quality $(\beta=0.01, P=0.556)$, sleep latency $(\beta=-0.01$, $P=0.481)$, sleep period $(\beta<-0.01, P=0.808)$ and number of awakenings $(\beta<-0.01, P=0.807)$ were not significantly associated with prior negative affect.

\section{Baseline subjective sleep and follow-up depressive symptoms}

With the exception of sleep latency, all sleep variables were significantly associated with follow-up depressive symptoms in the expected direction. Sleep quality as well as sleep period was negatively associated with future depressive symptoms $(\beta=-0.10, P<0.001$ and $\beta=-0.07, P=0.020)$. Number of awakenings was positively associated with future depressive symptoms at follow-up $(\beta=0.09, P=0.004)$. There was no significant association between sleep latency and future depressive symptoms $(\beta=0.02, P=0.535)$. In a sensitivity analysis, only including participants with no depressive symptoms at baseline on the SCL-90-R (scoring 1 'not at all' on all the items of the SCL-90-R depression subscale), the association between sleep quality and depressive symptoms at follow-up was significant $(\beta=-0.27, P<0.001)$.

Sleep variables that were significantly associated with SCL-90-R depressive symptoms at follow-up (sleep quality, number of awakenings, sleep period) were further analysed to examine whether they were also predictive for a DSM-IV diagnosis of depression at $T_{4}$. These analyses revealed that baseline sleep quality, number of awakenings and sleep period were not significantly associated with a diagnosis of major depression at follow-up (odds ratio $(\mathrm{OR}) 0.20(P=0.194), \mathrm{OR}=1.52(P=0.255)$ and $\mathrm{OR}=1.00$ $(P=0.736)$ respectively).

\section{Discussion}

The present study demonstrated a close association between subjective sleep and affect in a female population-based sample, both at the micro-level of daily associations as well as at the macro-level of longitudinal associations. Sleeping worse in terms of lower sleep quality and/or longer sleep latency was associated with individually reduced positive affect and increased negative affect the next day and was also predictive for the development of depressive symptomatology, even in a sample excluding participants with any experience of depressive symptomatology at baseline. Contrary to prior expectation, the association between sleep and affect did not appear to be bidirectional, as subjective sleep was associated with subsequent rather than prior affect. Thus, measures of sleep were good predictors of subsequent daytime affect, whereas measures of affect (with the exception of the association between positive affect and sleep quality, which may constitute a chance finding) did not predict subsequent sleep. Notably, negative affect did not have an impact on subsequent sleep.

\section{Uni- $\boldsymbol{v}$. bidirectional associations}

There appears to be only one other study that investigated the bidirectional associations between adult sleep and affect in daily life, however using a different approach. Talbot and colleagues ${ }^{6}$ examined the associations between total wake time during the night and daytime mood. They used a single-point affect measurement - that is, immediate pre-sleep mood and immediate post-sleep mood - and did not consider later or earlier periods during the day. In the present study, affect was prospectively and repeatedly sampled across the entire day using ESM. Furthermore, the sample of Talbot and colleagues was not population-based, but included patients with insomnia $(n=34)$, bipolar disorder $(n=49)$ and healthy controls $(n=52)$. In most of their analyses, group status moderated the effect between sleep and mood. In line with the present study, their results do not seem to support a bidirectional association between sleep and mood in healthy controls, but only in the two diagnostic groups. Furthermore, in accordance with the present results, it seemed that in healthy controls, sleep has more of an impact on positive affect than on negative affect the next day.

The finding of bidirectional associations between sleep and mood in the insomnia and bipolar groups in contrast to the healthy controls ${ }^{6}$ may be related to differences in sleep- and affect-related physiological and cognitive processes in healthy individuals compared with psychiatric populations. Harvey ${ }^{19}$ hypothesised a bidirectional link between sleep and affect in psychiatric disorders, as disturbance in mood may interfere with night-time sleep and sleep may interfere with daytime affect regulation, leading to a mutually maintaining process. Based on the current results it may be argued that in the general population, (perceived) night-time sleep may interfere with daytime affect regulation, but disturbance in mood is not great enough to lead to (perceived) night-time sleep interference.

The result that negative affect during the day does not appear to have an impact on subsequent sleep contrasts with prior expectations and a number of studies showing that pre-sleep affect influences sleep, ${ }^{20}$ even in healthy participants. However, most of these studies experimentally induced negative affect or stress, thus not representing the natural situation. The apparent lack of impact of prior negative affect on subjective sleep on a daily basis may be very important when considering the evolution of depression on a macro-level. That is, it seems that the subtle and repetitive impact of sleep on daily affect, rather than the subtle and repetitive impact of negative affect on sleep, may be one of the factors on the causal pathway to depression.

\section{Positive v. negative affect}

Negative and positive affect were differentially associated with sleep variables, as associations were stronger and more consistent 
for sleep and positive affect, in particular for sleep quality and positive affect. This finding is in line with recent investigations, all pointing towards a stronger role for positive affect in sleepaffect regulations. ${ }^{4,21-23}$ A possible explanation for this finding may lie in the construct of positive and negative affect. Positive affect is hypothesised to be a reward-oriented motivational system, attracting the individual to engage with the environment, whereas negative affect is hypothesised to represent the 'threat system', which reacts in response to hazards. ${ }^{24,25}$ In contrast to negative affect, positive affect is found to be subject to circadian rhythmicity throughout the 24-hour cycle, being highest during the afternoon and lowest during the second half of the night, possibly priming the individual for activity during daytime when the probability of reward is high. Negative affect is not found to demonstrate such rhythmicity, possibly as the individual has to be able to react to an (unpredictable) threat at any time. ${ }^{25-27}$ Given these findings, a stronger modulation of positive affect may be anticipated, ${ }^{4}$ possibly via interactions of circadian and sleep-wake dependent processes. ${ }^{28}$

It has been shown that the experience of positive affect can act as a buffer against negative reactivity and may thereby enhance emotional resilience against depression. ${ }^{29,30}$ Based on the data at hand, it can be argued that sleep disturbances may reduce the experience of positive affect in daily life, leading to an increased negative reactivity and enhanced probability of developing depression in the longer term. However, more research is needed to unravel the precise mechanisms by which sleep may affect the regulation of affect.

\section{Clinical significance}

The associations between subjective sleep and affect in daily life, as well as the associations between baseline sleep and follow-up depressive symptomatology using a dimensional scale, were statistically significant. There was no significant association between baseline sleep and the dichotomous measure 'DSM-IV diagnosis of depression at last follow-up'. The difference in the results between the dimensional measure of depressive symptoms (based on SCL-90-R) and the dichotomous measure of the presence of a diagnosis of depression (based on the SCID-I) may be power-related. The SCID-I was available for fewer participants than the SCL-90-R and is, in contrast to the SCL-90-R, only administered at one time point $\left(T_{4}\right)$. Furthermore, the present sample is not a clinical sample, but constitutes a population-based sample of relatively healthy females. The SCL-90-R may be a more sensitive instrument for the present population than the SCID-I.

Generally, effect sizes of around 0.2 are considered relevant but low, and those around 0.8 high. ${ }^{31}$ In the current study, effect sizes were small. However, the results from the ESM study were derived from data reflecting average increase or decrease in positive/ negative affect at repeated moments in daily life, thus representing a daily repetitive pattern of positive/negative affect change in association with a person's subjective sleep during each single day. Therefore, effect sizes reported in this study, although very small, cumulatively may well be clinically relevant.

\section{Limitations}

Some methodological limitations are apparent. Most important, sleep was not assessed objectively by means of polysomnography, but by self-reports, leading to two possible concerns. First, extended research has focused on the potential divergence of subjective and objective sleep data. ${ }^{32}$ This showed that generalisation from subjective to objective sleep is not necessarily possible. However, inaccurate self-report of sleep variables appears to be especially present in patients with disturbed sleep or patients with depression, ${ }^{32,33}$ whereas the current study focused on healthy individuals. Furthermore, the self-monitoring of sleep by means of a sleep diary can be regarded as one particular perspective of sleep, which does not necessarily agree with objective measurements, but which is nonetheless an important perspective. The diagnosis of insomnia, for instance, is based on reports of subjective, and not necessarily objective, sleep difficulties. Second, as with all self-reports, mood-congruent bias (i.e. mood on awakening may colour the sleep quality rating) or a negative appraisal style that explains the association between sleep and depressive symptoms (rather than sleep per se) cannot be conclusively ruled out. Future studies should include objective methods that can complement and validate self-report sleep data (i.e. by including actigraphy). Nevertheless, the association between subjective sleep and affect and subjective sleep and depression is clinically as important as the association between objective sleep and affect.

Furthermore, it has been suggested that problems may arise in the ESM procedure as it depends on the compliance of participants. ${ }^{34,35}$ During the collection of the data, no electronic devices were readily available to substitute the paper-and-pencil diary method. However, compliance levels were investigated in a subsample of the present sample, where participants were asked to take, during the ESM procedure, saliva samples at each of the ten unpredictable moments during the five consecutive days. ${ }^{10}$ Participants recorded collection times, unaware that compliance with the sampling protocol was being investigated by means of electronic monitoring devices. Results showed that adherence was high (over 90\%) and inclusion of the inaccurately timed samples did not distort the data. ${ }^{10}$ Therefore, results from the ESM procedure in this report can be considered valid.

Finally, the sample consisted of relatively highly educated, female participants. Since sleep, ${ }^{36,37}$ as well as its relation to affect, ${ }^{38}$ has been found to differ by gender and socioeconomic status, generalisation of the present results to male or lowereducated samples is not necessarily possible. However, one study focusing on the association between affect and sleep ${ }^{39}$ could not identify gender differences.

To conclude, the present study may shed light on the mechanisms by which sleep may be related to depression in women by investigating the association between subjective sleep and affect at a momentary day-to-day (micro) level. The subtle, repetitive impact of sleep on affect on a daily basis, especially on positive affect, may be one of the factors on the pathway to depression.

\footnotetext{
Jessica A. de Wild-Hartmann, MSC, Department of Psychiatry and Psychology, School of Mental Health and Neuroscience, Maastricht University Medical Centre Maastricht, and GGzE, Institute of Mental Health Care Eindhoven en de Kempen, Maastricht, and GGzE, Institute of Mental Health Care Eindhoven en de Kempen,
Eindhoven, The Netherlands; Marieke Wichers, PhD, Department of Psychiatry and Psychology, School of Mental Health and Neuroscience, Maastricht University Medical Centre, Maastricht, The Netherlands; Alex L. van Bemmel, MD, PhD, Department of Psychiatry and Psychology, School of Mental Health and Neuroscience, Maastricht University Medical Centre, Maastricht, and GGzE, Institute of Mental Health Care Eindhoven en de Kempen, Eindhoven, The Netherlands; Catherine Derom, PhD, Cindhove en derom, PhD, Centre of Human Genetics, University Hospital Leuven, and Department of Human Genetics, KU Leuven, Belgium; Evert Thiery, MD, PhD, Department of Neurology, Ghent University Hospital, Belgium; Nele Jacobs, PhD, Department of Psychiatry and Psychology, School of Mental Health and Neuroscience, Maastricht University Medical Centre, Maastricht, and Department of Psychology, Open University of the Netherlands, Heerlen, The Netherlands; Jim van Os, MD, PhD, Department of Psychiatry and Psychology, School of Mental Health and Neuroscience, Maastricht University Medical Centre, Maastricht, The Netherlands, and King's College, King's Health Partners, Department of Psychosis Studies, Institute of Psychiatry, London, UK. Health Partners, Department of Psychosis Studies, Institute of Psychiatry, London, UK
claudia J. P. Simons, PhD, Department of Psychiatry and Psychology, School of Mental Health and Neuroscience, Maastricht University Medical Centre, Maastricht, and GGzE, Institute of Mental Health Care Eindhoven en de Kempen, Eindhoven, The Netherlands
}

Correspondence: Jessica A. de Wild-Hartmann, Department of Psychiatry and Psychology, Maastricht University, PO Box 616, 6200 MD Maastricht, The Netherlands. Email: j.dewild-hartmann@maastrichtuniversity.nl

First received 27 Jun 2012, final revision 15 Nov 2012, accepted 4 Mar 2013 


\section{Funding}

This research was supported by the Netherlands Organisation for Scientific Research; the Fund for Scientific Research, Flanders and Twins, a non-profit association for scientific research in multiple births (Belgium) (to the East-Flanders Prospective Survey). M.W. was supported by the Netherlands Organisation for Scientific Research (NWO, VENI Grant No. 916.76.147) and by ZON-MW (Grant No. 171001002)

\section{Acknowledgement}

We thank all twins for their cooperation.

\section{References}

1 Ohayon MM, Roth T. Place of chronic insomnia in the course of depressive and anxiety disorders. J Psychiatr Res 2003; 37: 9-15.

2 Cho HJ, Lavretsky H, Olmstead R, Levin MJ, Oxman MN, Irwin MR. Sleep disturbance and depression recurrence in community-dwelling older adults: a prospective study. Am J Psychiatry 2008; 165: 1543-50.

3 Baglioni C, Battagliese G, Feige B, Spiegelhalder K, Nissen C, Voderholzer U et al. Insomnia as a predictor of depression: a meta-analytic evaluation of longitudinal epidemiological studies. J Affect Disord 2011; 135: 10-9.

4 Bower B, Bylsma LM, Morris BH, Rottenberg J. Poor reported sleep quality predicts low positive affect in daily life among healthy and mood-disordered persons. J Sleep Res 2010; 19: 323-32.

5 Zohar D, Tzischinsky O, Epstein R, Lavie P. The effects of sleep loss on medical residents' emotional reactions to work events: a cognitive-energy model. Sleep 2005; 28: 47-54.

6 Talbot LS, Stone S, Gruber J, Hairston IS, Eidelman P, Harvey AG. A test of the bidirectional association between sleep and mood in bipolar disorder and insomnia. J Abnorm Psychol 2012; 121: 39-50.

7 Cousins JC, Whalen DJ, Dahl RE, Forbes EE, Olivio TM, Ryan ND, et al. The bidirectional association between daytime affect and nighttime sleep in youth with anxiety and depression. J Pediatr Psychol 2011; 36: 369-79.

8 Derom CA, Vlietinck RF, Thiery EW, Leroy FO, Fryns JP, Derom RM. The East Flanders Prospective Twin Survey (EFPTS). Twin Res Hum Genet 2006; 9 : 733-8.

9 Loos R, Derom C, Vlietinck R, Derom R. The East Flanders Prospective Twin Survey (Belgium): a population-based register. Twin Res 1998; 1: 167-75.

10 Jacobs N, Nicolson NA, Derom C, Delespaul P, van Os J, Myin-Germeys I. Electronic monitoring of salivary cortisol sampling compliance in daily life. Life Sci 2005; 76: 2431-43.

11 Kendler KS, Thornton LM, Prescott CA. Gender differences in the rates of exposure to stressful life events and sensitivity to their depressogenic effects. Am J Psychiatry 2001; 158: 587-93.

12 Csikszentmihalyi $M$, Larson R. Validity and reliability of the ExperienceSampling Method. J Nerv Ment Dis 1987; 175: 526-36.

13 Delespaul P. Assessing Schizophrenia in Daily Life: The Experience Sampling Method. Maastricht University Press, 1995.

14 Carney CE, Buysse DJ, Ancoli-Israel S, Edinger JD, Krystal AD, Lichstein KL, et al. The consensus sleep diary: standardizing prospective sleep selfmonitoring. Sleep 2012; 35: 287-302.

15 First MB, Gibbon M, Spitzer RL, Williams JB. Structured Clinical Interview for DSM-IV-TR Axis I Disorders, Research Version, Patient Edition (SCID-I/P). Biometrics Research, New York State Psychiatric Institute, 2002.

16 Derogatis LR, Rickels K, Rock AF. The SCL-90 and the MMPI: a step in the validation of a new self-report scale. Br J Psychiatry 1976; 128: 280-9.

17 Eysenck H, Eysenck S. Manual of the Eysenck Personality Scales. Hodder \& Stoughton, 1991.
18 Snijders T, Bosker R. Multilevel Analysis: An Introduction to Basic and Advanced Multilevel Modeling. SAGE publications, 1999

19 Harvey AG. Insomnia, psychiatric disorders, and the transdiagnostic perspective. Curr Dir Psychol Sci 2008; 17: 299-303.

20 Vandekerckhove M, Weiss R, Schotte C, Exadaktylos V, Haex B, Verbraecken $\mathrm{J}$, et al. The role of presleep negative emotion in sleep physiology. Psychophysiology 2011; 48: 1738-44.

21 Paterson JL, Dorrian J, Ferguson SA, Jay SM, Lamond N, Murphy PJ, et al. Changes in structural aspects of mood during 39-66 $\mathrm{h}$ of sleep loss using matched controls. Appl Ergon 2011; 42: 196-201.

22 Dagys N, McGlinchey EL, Talbot LS, Kaplan KA, Dahl RE, Harvey AG. Double trouble? The effects of sleep deprivation and chronotype on adolescent affect. J Child Psychol Psychiatry 2011; 53: 660-7.

23 Talbot LS, McGlinchey EL, Kaplan KA, Dahl RE, Harvey AG. Sleep deprivation in adolescents and adults: changes in affect. Emotion 2010; 10: 831-41.

24 Murray G, Allen NB, Trinder J. Mood and the circadian system: investigation of a circadian component in positive affect. Chronobiol Int 2002; 19 $1151-69$.

25 Murray G, Nicholas CL, Kleiman J, Dwyer R, Carrington MJ, Allen NB, et al. Nature's clocks and human mood: the circadian system modulates reward motivation. Emotion 2009; 9: 705-16.

26 Clark LA, Watson D, Leeka J. Diurnal variation in the positive affects. Motiv Emot 1989; 13: 205-34.

27 Watson D, Wiese D, Vaidya J, Tellegen A. The two general activation systems of affect: Structural findings, evolutionary considerations, and psychobiological evidence. J Pers Soc Psychol 1999; 76: 820-38.

28 Boivin DB, Czeisler CA, Dijk DJ, Duffy JF, Folkard S, Minors DS, et al. Complex interaction of the sleep-wake cycle and circadian phase modulates mood in healthy subjects. Arch Gen Psychiatry 1997; 54: 145-52.

29 Wichers M, Jacobs N, Derom C, Thiery EW, van Os J. Depression: too much negative affect or too little positive affect? Twin Res 2007; 10: 19-20.

30 Wichers MC, Myin-Germeys I, Jacobs N, Peeters F, Kenis G, Derom C, et al. Evidence that moment-to-moment variation in positive emotions buffer genetic risk for depression: a momentary assessment twin study. Acta Psychiatr Scand 2007; 115: 451-7.

31 Cohen J. Statistical Power Analysis for the Behavioral Sciences (2nd edn). Erlbaum, 1988.

32 Armitage R, Trivedi M, Hoffmann R, Rush AJ. Relationship between objective and subjective sleep measures in depressed patients and healthy controls. Depress Anxiety 1997; 5: 97-102.

33 Tang NK, Harvey AG. Altering misperception of sleep in insomnia: behavioral experiment versus verbal feedback. J Consult Clin Psychol 2006; 74: 767-76.

34 Broderick JE, Arnold D, Kudielka BM, Kirschbaum C. Salivary cortisol sampling compliance: comparison of patients and healthy volunteers. Psychoneuroendocrinology 2004; 29: 636-50.

35 Kudielka BM, Broderick JE, Kirschbaum C. Compliance with saliva sampling protocols: electronic monitoring reveals invalid cortisol daytime profiles in noncompliant subjects. Psychosom Med 2003; 65: 313-9.

36 Grandner MA, Patel NP, Gehrman PR, Xie D, Sha D, Weaver T, et al. Who gets the best sleep? Ethnic and socioeconomic factors related to sleep complaints. Sleep Med 2010; 11: 470-8.

37 Krishnan V, Collop NA. Gender differences in sleep disorders. Curr Opin Pulm Med 2006; 12: 383-9.

38 Berry DT, Webb WB. State measures and sleep stages. Psychol Rep 1983; 52: 807-12.

39 McCrae CS, McNamara JP, Rowe MA, Dzierzewski JM, Dirk J, Marsiske M, et al. Sleep and affect in older adults: using multilevel modeling to examine daily associations. J Sleep Res 2008; 17: 42-53. 